\title{
Percepções de estudantes de Odontologia sobre a experiência em um estágio não obrigatório no SUS
}

\author{
Soraya Fernandes Mestriner*; Luana Pinho de Mesquita**; Wilson Mestriner Júnior*; Alexandre \\ Fávero Bulgarelli***
}

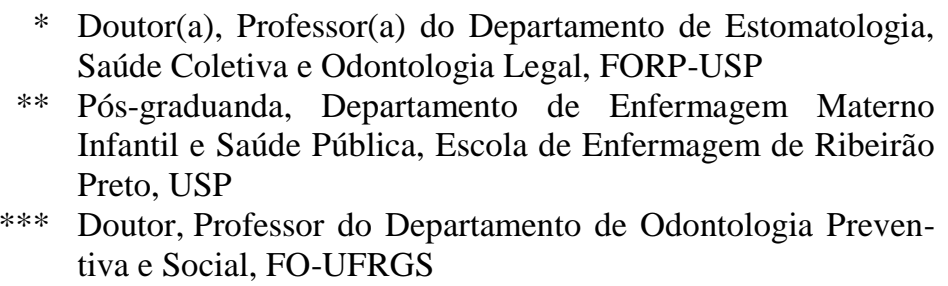

Recebido em 02/05/2017. Aprovado em 25/09/2017.

\begin{abstract}
RESUMO
O estudo teve por objetivo analisar as percepções de estudantes de Odontologia sobre a experiência em um estágio não obrigatório no sistema público de saúde brasileiro em um município de São Paulo e sua contribuição na formação profissional. Trata-se de um estudo descritivo, de natureza qualitativa com estudantes de uma universidade pública, no período de 2013-2015. Foram realizadas entrevistas semidirigidas que foram gravadas e transcritas. Os dados foram sistematizados e analisados por meio da técnica da Análise de Conteúdo na perspectiva temática com suporte teórico das Diretrizes Curriculares Nacionais para o Curso de Odontologia. Observou-se que os estudantes perceberam o estágio como uma oportunidade para vivenciar a realidade do trabalho nos serviços de saúde, desenvolveram e aperfeiçoaram habilidades e competências como comunicação, gerenciamento e organização de seu trabalho e construção de sua identidade profissional. Conclui-se que os estudantes se aproximaram dos conceitos de atenção à saúde, humanização, autonomia e aprendizagem no trabalho, experiência que se mostrou significativa.
\end{abstract}

Descritores: Educação em Odontologia. Estágios. Serviços de Saúde. Humanização da Assistência.

\section{INTRODUÇÃO}

A formação de profissionais de saúde no Brasil, como em outros países, passou a ser objeto de análise e reflexão no sentido de possibilitar respostas às necessidades de saúde da sociedade $^{1}$. A integração teoria-prática, o trabalho multiprofissional e em equipe, a integralidade do cuidado em saúde e a formação de vínculo, na perspectiva da clínica ampliada, vêm potencializando a reorganização do modelo de cuidado e a formação em saúde ${ }^{2,3}$. A articulação entre universidades e serviços de saúde mostra-se uma relevante estratégia para o alcance da integralidade do cuidado tendo em vista que é necessário superar os reducionismos do modelo biomédico e possibilitar o exercício do olhar ampliado para as 
necessidades de saúde ${ }^{4,5}$.

Neste sentido, no Brasil, políticas públicas têm fomentado parcerias e articulações entre a academia, os serviços de saúde e/ou a comunidade, para o enfrentamento das necessidades de saúde da sociedade, com foco nas atividades de ensino, extensão e pesquisa ${ }^{6-8}$. Deste modo, evidencia-se cada vez mais o estímulo ao diálogo na interface ensino-serviço-comunidade em forma de redes colaborativas que propõem a vivência em campos de aprendizagem ${ }^{9}$.

As experiências em novos cenários de prática, como parte do processo ensino-aprendizagem em Odontologia, podem ser importantes instrumentos para formação de profissionais integrados com as realidades sociais e articulados com os desafios na atenção à saúde bucal brasileira ${ }^{10}$. O estágio é o instrumento de integração e conhecimento do estudante com a realidade social e econômica de sua região e do trabalho de sua área; também, deve ser entendido como o atendimento integral ao usuário que o estudante de Odontologia presta à comunidade, intra e extramuros; deve fomentar a relação ensino-serviços e ampliar as relações da Universidade com a Sociedade ${ }^{11}$. Deste modo, o estudante passa a compreender a reorganização do processo de trabalho no Sistema Único de Saúde (SUS) para construir um olhar mais ampliado do seu "objeto de trabalho" que é o usuário do serviço ${ }^{2,3}$. A ampliação de espaços de aprendizagem e a exploração de novas formas de parcerias entre as instituições de ensino e diferentes setores da sociedade contribuem para a formação de estudantes de Odontologia.

Considerando a importância da ampliação da inserção dos estudantes em cenários de prática no SUS e as possíveis transformações destas vivências para sua formação, o objetivo desse estudo foi analisar as percepções de estudantes de Odontologia sobre a experiência em um estágio não obrigatório no SUS em um município de São Paulo, Brasil, e sua contribuição na formação profissional.

\section{MÉTODO}

Estudo descritivo de natureza qualitativa, desenvolvido por meio da construção de categorias temáticas emergentes da análise das percepções presentes nas falas dos participantes. Tratase de um estudo de significados, valores e atitudes dos processos e dos fenômenos que não podem ser quantificados e cuja vivência pode se expressar na linguagem ${ }^{13}$. Dessa forma, neste estudo, valorizou-se a percepção da experiência vivida e apresentada na fala ${ }^{14}$, e o conhecimento adquirido com a vivência.

A Faculdade de Odontologia de Ribeirão Preto da Universidade de São Paulo (FORPUSP), em sua reestruturação curricular implantada em 2004, ampliou seus cenários de prática, possibilitando a aproximação com os serviços de saúde do SUS, com atividades de atenção à saúde junto a equipes da Estratégia Saúde da Família (ESF) do distrito Oeste de saúde do município de Ribeirão Preto, desde o primeiro ano, com duração e complexidade crescentes. Na perspectiva de ampliar ainda mais os cenários de práticas, a partir de 2007, esta IES firmou convênios ${ }^{15,16} \mathrm{com}$ a Prefeitura Municipal de Sertãozinho, para estágio não obrigatório no SUS. O referido município está localizado na Região Metropolitana de Ribeirão Preto a uma distância de $21 \mathrm{~km}$ da Universidade.

O ingresso dos estudantes no estágio, cenário da presente pesquisa, se dá por processo seletivo estabelecido em edital, com análise curricular e documental. Foram atribuídos pontos para a referida análise documental, nos quesitos: participações em monitorias, eventos (cursos, congressos, simpósios, apresentação de trabalhos científicos) e projetos de pesquisa e extensão re 
lacionados à rede de atenção à saúde. Em caso de empate na pontuação final, o desempate era feito pela maior média ponderada das disciplinas cursadas. O estágio tem duração de até 12 meses, com bolsa oferecida pela concedente (Prefeitura Municipal de Sertãozinho) pela jornada de 20 horas semanais.

As atividades de estágio são realizadas de segunda a sábado e estão previstas em seu Plano Individual de Trabalho, fundamentadas nos princípios e diretrizes do SUS, na Política Nacional de Saúde Bucal ${ }^{17}$ e nas Diretrizes Curriculares Nacionais (DCN) para os Cursos de Odontologia $^{18}$. Dentre elas estão: territorialização com fins de caracterização da população e de seus problemas de saúde; diagnóstico em saúde bucal; planejamento das ações de saúde bucal levando em consideração o contexto local, capacidade instalada e perfil epidemiológico da população em questão; ações coletivas de educação em saúde, escovação supervisionada, fluorterapia, e procedimentos individuais cirúrgico-restauradores nas Unidades Básicas de Saúde, Centro de Especialidades Odontológicas (CEO) e espaços sociais vinculados à Prefeitura Municipal de Sertãozinho. As atividades de planejamento, estudo e discussão de casos, confecção de relatórios e avaliação podem ser realizadas na IES, em horários compatíveis com a escala de atividades do estagiário.

O acompanhamento e avaliação são realizados por profissionais cirurgiões-dentistas da rede municipal de saúde de Sertãozinho, e docentes da área de Saúde Coletiva da IES. Estes atuam como tutores de campo, supervisionando e orientando as atividades dos estudantes. Existe uma comunicação direta dos tutores com os preceptores de maneira que haja construção conjunta das atividades.

Participaram deste estudo nove estudantes do curso de Graduação em Odontologia da FORP-USP, referente ao total de estudantes que realizaram o estágio no período estudado. Em sua maioria do sexo feminino, com idade média de 22 anos, vivenciaram em média doze meses o estágio não obrigatório no período de 2013-2015.

Foram realizadas entrevistas semidirigidas por meio de um roteiro, que foram gravadas e posteriormente transcritas ${ }^{19}$. Tais entrevistas aconteceram em ambientes sem interferências de ruídos externos, conversas e fluxo de outras pessoas. Estas situações foram pensadas, pois têm um potencial para desviar o interesse do entrevistado no momento da entrevista. Pensou-se, também, em local com privacidade para conforto do estudante no momento da sua fala. Os assuntos abordados no roteiro norteador foram embasados em perguntas que motivaram conversas entre o pesquisador e o estudante sobre a macro questão da pesquisa.

A análise dos dados foi realizada pela técnica de Análise de Conteúdo temática ${ }^{20}$. Os pesquisadores seguiram as seguintes etapas de análise: pré-análise, exploração do material, tratamento dos dados e interpretação. A construção dos resultados foi norteada pelo referencial das DCN para os cursos de Odontologia ${ }^{21}$ a saber: atenção à saúde, tomada de decisões, comunicação, liderança, administração e gerenciamento e educação permanente. Deste modo foram construídos dois temas: A transformação da prática; e A construção de novos sentidos para a formação do cirurgião dentista.

No processo de análise, surgiram hipóteses como: o estudante encontra nesta experiência aspectos presentes na DCN; o estudante percebe que tal experiência permite situações que enriquecem sua formação; o estudante constrói novos sentidos para sua formação. Foram então identificados temas e núcleos de sentidos da percepção dos estudantes. De acordo com a técnica adotada, tratar o material significa codificá-lo 
com objetivo de descrever o conteúdo das mensagens e assim, a partir dos núcleos de sentido, foram eleitas unidades de registro seguidas de unidades de contexto.

Este estudo teve aprovação do Comitê de Ética em Pesquisa. Os estudantes que por livre vontade decidiram contribuir e assinaram os Termos de Consentimento Livre e Esclarecido (TCLE) conforme explicitado no capítulo IV da Resolução CNS $466 / 12^{22}$.

\section{RESULTADOS E DISCUSSÃO}

A vivência no estágio não obrigatório no SUS revelou um processo de aprendizagem em que o estudante mostra interesse em participar de uma experiência fora da universidade e, portanto, não realiza um estágio compulsoriamente. Neste caso o estudante busca, por livre vontade, ampliar suas experiências no serviço público de saúde. Acredita-se que tal interesse é resultado de motivações durante a formação. Trata-se de uma experiência diferente daquela obrigatória do currículo.

O fato de ser um estágio não obrigatório gera curiosidade e estímulo que se refletem em motivação para conhecer cenários de prática diferentes. $\mathrm{O}$ presente estudo mostra que o referido estágio apresenta novos desafios e diferentes elementos no processo de trabalho e humanização das práticas. Portanto, essa experiência é apontada como capaz de motivar o estudante a refletir sobre questões sociais que permeiam a odontologia, além de estimular as práticas extramurais ${ }^{23}$.

O estágio extramuros em questão não faz parte da matriz curricular obrigatória da graduação. Ele constitui-se como um projeto de extensão em que os estudantes se inscrevem e passam por uma seleção. Outro fato importante a ser destacado é a participação dos estudantes em atividades na Atenção Primária à Saúde (APS) em uma Unidade Básica de Saúde (UBS) e na atenção secundária, no CEO, onde eles têm aproximação à dinâmica dos diferentes serviços e convivência com os profissionais que nela trabalham. Inclui-se na programação do estágio a participação em ações de promoção e prevenção em espaços sociais, com a interação com estudantes da educação infantil, ensino fundamental, professores e gestores.

A vivência nos serviços de saúde do SUS, seja na APS ou em ambientes hospitalares, é reconhecida como uma rica oportunidade de aprendizado para os estudantes $^{24,25}$. Nestes casos há uma real aproximação com os desafios, limites e possibilidades de trabalho nestes ambientes ao vivenciarem na prática alguns dos princípios do SUS e valorizarem o serviço público em seu processo de formação ${ }^{26}$. A escolha por dados qualitativos para a exploração das percepções dos estudantes nestas experiências de integração ensino-serviço-comunidade constitui-se como abordagem importante para o entendimento do olhar dos estudantes para as mudanças necessárias em sua formação ${ }^{27}$.

Aspectos das DCN para o curso de Odontologia, suporte teórico utilizado para a análise da percepção dos entrevistados, são evidenciados em suas falas. Assim, a análise destes dados possibilitou a exploração de duas categorias temáticas na construção do conhecimento dos estudantes: "transformando a minha prática" e "construindo novos sentidos para minha formação em saúde".

\section{Transformando minha prática}

O tema sobre a transformação da prática que o estudante adquiriu até o momento em que vivenciou o estágio reflete as premissas apresentadas pelas DCN que envolvem elementos do processo de trabalho, habilidades, competências e sentidos que foram desenvol- 
vidos e/ou aperfeiçoados pelos estudantes durante o estágio não obrigatório. Ressalta-se que possibilitar espaços para vivenciar conceitos da saúde coletiva são grandes sensibilizadores de futuros profissionais preocupados e reflexivos sobre a saúde das comunidades e seus determinantes $^{28,29}$.

A adaptação ao ambiente de trabalho em um município diferente e a realidade do trabalho trouxe certa insegurança do início e a vivência da superação dos obstáculos:

“( ) no começo dava bastante medo mas depois a gente foi pegando a prática” E2

Pesquisa sobre a percepção dos estudantes de Odontologia na atenção básica ${ }^{11}$, demonstrou que essa adaptação gradativa é essencial não só para o desenvolvimento de habilidades clínicas mas para a compreensão do contexto da comunidade na qual se inserem, corroborando o fato de que esta vivência possibilita o desenvolvimento de diferentes graus de autonomia ao longo do processo de trabalho e o reconhecimento de suas conquistas $^{26}$.

A experiência da tomada de decisões no cotidiano do trabalho, com apoio da preceptoria e incentivo à autonomia, foi evidenciada como uma diferença da vivência na instituição de ensino:

"tomava uma decisão muitas vezes juntos (o estagiário e a preceptora) (...) diferente do aprendido na faculdade (...) a gente buscava uma solução juntos" E3.

Outro estudante (E5) afirmou que "tentava raciocinar" e muitas vezes, também, esclarecia suas dúvidas com a preceptora. O estudante, desta forma, percebe que pôde praticar a tomada de decisão autônoma em parceria com o preceptor, que teve um papel de apoio importante para o desenvolvimento desta habilidade, pois se mostrava disponível a discutir os casos e as possibili- dades em cada situação. O estudante se sente seguro pela postura de proximidade, disponibilidade e paciência do preceptor que tem um papel de articulador das ações e atividades no território $^{30}$.

Além disso, o estágio foi visto como um ambiente que apresenta os desafios típicos do cotidiano do trabalho, com a possibilidade de conhecer outras formas de gerenciar o trabalho em Odontologia:

"tratar casos de maneiras diferentes tendo um resultado positivo" E1. "às vezes não tinha rolete de algodão aí chegava o rolete de algodão e acabava a gaze... aí acabava a gaze e não tinha luva $P P(. .$.$) sei que é parte do serviço público$ né? a gente sabe..." $\mathrm{E} 9$.

“(...) assim...na verdade a gente não tem assim muito tempo pra conversar com o paciente... (... )' $\mathrm{E} 4$.

A possibilidade de vivenciar a gestão do trabalho e dos serviços de saúde a partir da administração e gerenciamento de seu próprio trabalho e do trabalho com a equipe de saúde bucal, também foi percebida pelos estudantes. Vale ressaltar que o trabalho em equipe aprimora a qualidade de um serviço de saúde e também a qualidade da formação profissional ${ }^{2,3,31}$. Tal pressuposto é identificado na percepção dos estudantes como a seguir:

"aprendi a ter mais habilidade para diagnosticar e fazer plano de tratamento" E5.

“atendia todas as urgências (...) depois começava o atendimento dos agendados (...) (se tinha) alguma dúvida de certa especialidade (...) perguntava para o dentista (do serviço de saúde) que tinha uma experiência com aquele tipo de tratamento" E7 
Percebe-se o desenvolvimento e aperfeiçoamento de habilidades de planejamento, respeito às necessidades do usuário, comunicação em equipe e enfrentamento de obstáculos e imprevistos no cotidiano dos serviços, como a falta de material e o grande número de atendimentos. Neste processo o estudante se permite trabalhar com o outro e cultiva a arte do encontro ${ }^{14}$, e deste modo aprende a integrar o outro profissional na dinâmica do trabalho, sendo fundamental o princípio da comunicação. Na percepção dos estudantes há o reconhecimento da importância da comunicação identificada por ações de humanização do cuidado prestado aos usuários. A humanização na assistência está diretamente associada à compreensão de que a clínica ampliada é o caminho para uma reorganização do serviço de maneira a refletir na qualidade do cuidado ao usuário do serviço de saúde ${ }^{32}$. Vê-se que houve o aprendizado e cuidado com a linguagem com diferentes idades, como com idosos e crianças e com usuários de diferentes situações socioeconômicas, demonstrando o desenvolvimento de senso crítico e sensibilidade em favor da autonomia do usuário:

"Pela carência da população (...) sempre tentava comunicar da melhor forma possível porque muitos ficavam assustados com medo (...) aprendi mais humildade (...) dar mais atenção (...) olhar para o paciente e ver que ele está precisando não só do tratamento mas de uma ajuda psicológica também” $\mathrm{E} 3$

A comunicação é algo relevante para a formação de profissionais da saúde. É na graduação que existe o espaço para o desenvolvimento e o treinamento de habilidades para se comunicar. Boa comunicação leva à construção de boas relações interpessoais bem como à troca de informação e de experiências com o usuário e com os colegas de trabalho, aspectos fundamentais para a formação de cirurgiões-dentistas ${ }^{33}$. O processo de análise dos dados permitiu a compressão de que o estudante transforma percepções pré-existentes sobre sua prática ampliando sua clínica e visão sobre o cuidado em saúde bucal. Tal transformação é reflexo da possibilidade de ampliar experiências, fora dos muros da Universidade, por livre escolha. Deste modo o estudante abre caminho, a partir desta experiência vivida com o estágio, para construir novos sentidos para sua formação.

\section{Construindo novos sentidos para minha for- mação em saúde}

Publicações sobre mudanças curriculares nos cursos de Odontologia ressaltam a persistência do modelo tradicional no Brasil com valorização da prática odontológica de cunho libe$\mathrm{ral}^{34,35}$, o que pode determinar certa resistência ou rejeição dos estudantes às práticas extramuros nos serviços de saúde pública, ou à sua inserção de trabalho no SUS, ou ainda, o não reconhecimento como prática inovadora e necessária ${ }^{35}$.

A proposta de integração do profissional odontólogo ao SUS vai ao encontro dos investimentos federais no fortalecimento da APS, com a inserção de equipes de saúde bucal na ESF. Porém, entende-se que estas transformações são processuais e que este fator ainda não é determinante para a mudança curricular imediata nas IES, outrossim, elas exigem tempo e avaliação permanente dos currículos ${ }^{34}$.

Há a sinalização para uma mudança paradigmática na formação de um profissional crítico, capaz de aprender a aprender, de trabalhar em equipe e de levar em conta a realidade social $^{36}$. Na perspectiva de alcançar o perfil do egresso proposto para os cursos de Odontologia, são recomendadas habilidades e competências gerais definidas nas DCN e sintetizadas como: 1) atenção à saúde: pensar criticamente e analisar problemas da sociedade e procurar soluções; 2) 
tomada de decisão; 3) comunicação: interação com demais profissionais e com a população; 4) liderança: para atuar em equipe multiprofissional; 5) administração e gerenciamento: iniciativa e empreendedorismo nas equipes de saúde; 6) educação permanente: aprender continuamente $^{37}$.

No eixo temático "Construindo novos sentidos em minha formação em saúde" exploraramse aspectos das DCN que ainda apresentam certa fragilidade nas falas dos estudantes, são aspectos do aprendizado que estão em construção. Os estudantes apresentaram possibilidades de descobrir caminhos profissionais; apontaram limites e desafios em relação à liderança. Destaca-se a necessidade de uma reflexão crítica acerca de conceitos como Educação Permanente em Saúde e Atenção à Saúde no aprendizado destes estudantes.

A experiência de viver o cotidiano dos serviços de saúde foi fator determinante para a segurança da escolha profissional, uma oportunidade para construírem sua identidade profissional.

No entanto, o perfil profissional do odontólogo na atualidade ainda tem um caráter muito individualista e suas preocupações mais evidentes são em relação ao mercado de trabalho considerado competitivo e a necessidade de dominar as técnicas e procedimentos ${ }^{24}$. É importante observar que no contexto do descobrimento de outras possibilidades de atuação na saúde vem a descoberta da atuação na saúde das comunidades. Este interesse vem crescendo na formação em Odontologia. Tal contexto vem ao encontro do presente estudo pois o referido estágio amplia as possibilidades de formação. Nos trechos a seguir, nota-se o quanto os estudantes valorizam o aprendizado da vivência:

"a melhor experiência prática profissional (...) aprende muito (...) pratica muito"
E5.

"falta pouco tempo para a gente se formar e o mercado está cada vez mais competitivo (...) tudo o que você puder fazer para se preparar melhor é válido, pra mim foi muito gratificante aprendi muito" E9

Esses aspectos são constantes nas falas e percepções dos estudantes e corroboram resultados de pesquisas que abordam a necessidade de discussões sobre ética e sobre aperfeiçoamento humanístico na formação dos estudantes para que as diretrizes que orientam os currículos sejam integralmente colocadas em prática ${ }^{38}$.

A liderança foi um aspecto pouco discutido pelos estudantes nas entrevistas e muitas vezes foi confundida com pró-atividade e organização, deixando de lado os aspectos éticos e a responsabilidade coletiva exigidos de um profissional de saúde que trabalha em equipe.

“(...) então lá (no estágio) a gente abre muitas possibilidades de como executar nosso trabalho" E7

Por outro lado, a vivência nos serviços de saúde em um município diferente daquele que o estudante reside e para o qual foi necessário o entendimento do contexto de vida de seus moradores e aspectos da gestão do trabalho em saúde locais, foi oportunidade para diferenciar as realidades de trabalho e valorizar o espaço de ensinoaprendizagem extramuros (da Universidade e do próprio município):

"tive muito mais experiência (...) agilidade (...) percebi a diferença tendo contato com outros alunos que ainda não participavam (percebi) o que eu tinha aprendido" $\mathrm{E} 3$.

“(...) com o estágio a gente conseguia ver um outro aspecto do atendimento" E6.

"hoje a gente consegue resolver muita coisa que antes a gente não sabia como 
fazer (...) acho que foi muito importante. E7.

"aprendi a lidar com pacientes diferentes da faculdade em condições diferentes totalmente (...) tanto bucal quanto física $e$ economicamente" E5

A aproximação ao conceito de Atenção à Saúde por meio da experiência na realidade e de compreensão do usuário em seu contexto possibilitou o desenvolvimento de uma clínica mais ampliada sobre os determinantes de saúde e para a complexidade do processo saúde-doença, aproximando-se das características de um perfil esperado para um futuro trabalhador da APS, como o desenvolvimento de um olhar para além do biomédico, que envolve o usuário e sua família no plano de cuidados e contribui na humanização das práticas e no estímulo ao desenvolvimento da cidadania $^{32,39}$

A vivência da Atenção à Saúde esteve presente também nas atividades em espaços sociais como a escola, o que possibilitou a experiência da intersetorialidade e da comunicação com diferentes atores do território como professores e gestores. A promoção da intersetorialidade, por ser uma dimensão importante para a governança de um serviço de saúde, é preconizada aos profissionais de saúde em âmbito mundial e devem abrir espaço para a interação e colaboração entre diferentes setores ${ }^{37}$.

As atividades de educação em saúde realizadas nestes espaços possibilitaram o desenvolvimento da sensibilidade, da comunicação e do entendimento de possíveis resultados positivos destas ações coletivas:

"a gente fez a avaliação de risco (na escola) e também a gente fez teatrinho (...) foi bem legal porque elas ficavam muito motivadas (..) muitas (crianças) tinham classificação de urgência (...) foi bem interessante os teatrinhos" $\mathrm{E} 8$
A vivência da Educação Permanente na formação nos serviços foi evidenciada com a troca de experiências e saberes com a equipe das unidades de saúde, principalmente com aqueles considerados "mais experientes":

"você passa a aprender (...) trocar informações com outros profissionais (...) a trabalhar numa divisão de trabalhos" E3. "foi muito bom mesmo porque as vezes um dentista eles sabem muito de uma determinada área mas as vezes uma outra coisa ele não sabe (...) achei muito bom" E7

Houve a percepção de que a realidade dos serviços com alta demanda de atendimentos não abre espaço para momentos de reflexão, como no caso em que o estudante foi indagado se seria importante ter momentos de discussão em equipe:

"ah eu acho que não é tanto o foco (no estágio) eu acho que é importante sim (...) não acho necessário um momento de ter uma discussão de caso assim como se fosse no núcleo (Unidade de Saúde da Família) por exemplo" E9.

A formação nos serviços de saúde e o aprendizado no trabalho ainda não são reconhecidos como parte integrante do cotidiano para alguns destes estudantes. Este entendimento é confirmado nos serviços de saúde que são orientados por um modelo pouco integrado entre seus membros e no qual os profissionais trabalham de forma fragmentada e por isso entende que sua formação deveria ocorrer fora da unidade de saúde e não em seu cotidiano ${ }^{40}$.

Para Batista e Gonçalves ${ }^{41}$, o processo de qualificação dos profissionais de saúde deve ocorrer desde a graduação, abrindo possibilidades para a democratização dos espaços de trabalho, em um movimento de aprender e de ensinar entre todos os atores envolvidos e na busca por soluções criativas para os problemas encontrados 
em uma realidade complexa. Para isso é necessário que os docentes estejam abertos para a escuta dos estudantes e para a construção de um processo de aprendizagem que motive os estudantes a participar de forma contínua, despertando o seu interesse:

“(...) eu entendi um pouquinho como funciona o atendimento público né?" E3.

É portanto importante que professores estejam abertos para a escuta dos estudantes.

O desenvolvimento moral, crítico e ético diante do novo contexto pode aportar diferentes dimensões a serem destacadas dentre os conhecimentos vividos e apreendidos. A gestão do próprio trabalho de forma autônoma, segura e organizada e a comunicação com o usuário e com os demais profissionais foram reconhecidas pelos estudantes como fatores essenciais para o sucesso de futuros cirurgiões-dentistas.

Além disso, a condição de experiência extramuros, fora do ambiente seguro da Universidade, no qual os estudantes trabalharam aspectos relacionados às necessidades e ao contexto de saúde local foi importante para o entendimento da dimensão do conceito de saúde, de seus determinantes e condicionantes não só no sistema de saúde, mas, também, associando as atividades a espaços sociais, como a escola.

Ressalta-se que a percepção de preceptores e docentes sobre o processo ensino-aprendizagem em cenários como este é igualmente importante e necessária para o diálogo e para a reflexão sobre o processo de trabalho e também para garantir que a comunidade seja o centro e fim das ações de saúde.

\section{CONSIDERAÇÕES FINAIS}

A percepção dos estudantes em relação à sua vivência em cenários de prática, como explorado neste artigo, tem sido objeto de estudo recorrente na realidade brasileira, principalmente a partir das DCN, ainda em vigência, para os cursos da área da saúde e das premissas para a formação de profissionais de saúde que se comprometam com as necessidades de saúde do Brasil.

Dessa forma, nota-se que as percepções desses futuros profissionais em relação à sua experiência foram, em sua maioria, positivas e também consequência de uma aprendizagem significativa e processual. Aspectos negativos foram percebidos em relação à fase de adaptação inicial, e parte da compreensão do ambiente de trabalho que exige rapidez e agilidade no atendimento. Os estudantes consideraram o estágio não obrigatório como uma oportunidade importante para aprimorar e ampliar seus conhecimentos técnicos e conhecer a realidade dos serviços de saúde e do mundo do trabalho ao vivenciar o cotidiano do SUS na prática, o que exigiu gerenciamento e organização de seu trabalho e trouxe segurança para fazer escolhas e oportunidade de construção de sua identidade profissional.

Para além do manejo de técnicas, os estudantes perceberam transformações em sua postura como profissionais de saúde. Ao se depararem com o contexto e as necessidades da comunidade, se viram comprometidos com o bem-estar dos usuários e com a interação com a equipe de trabalho, demonstrando que a aprendizagem desenvolveu novos sentidos a partir da experiência vivida neste cenário. E, considera-se a possibilidade de futuras investigações que incluam também as percepções de preceptores e docentes nesta experiência de estágio.

Para finalizar, presume-se que além de possibilitar momentos reflexivos dos profissionais responsáveis pelo acompanhamento formativo dos estudantes neste estágio, este estudo resulte em valorização do saber da experiência dos atores envolvidos nesta parceria ensino-serviço-comunidade e apoio à ampliação de cenários de práticas e de aprendizagem que permitam transfor- 
mações na comunidade e nas práticas de profissionais de saúde comprometidos com a saúde integral e de qualidade.

\section{ABSTRACT \\ Perceptions of Dental students regarding their experiences in a non-mandatory internship in the Brazilian Unified Health System (SUS)}

This study aimed to analyze the perceptions of Dental undergraduate students about the experience in a non-mandatory internship in the Brazilian Public Health System, in a municipality of São Paulo, Brazil, and its contribution to professional education. We performed a descriptive study in a qualitative approach with undergraduate students of a public university, from 2013 to 2015. Semi-structured interviews were conducted, audio-recorded and transcribed. The data were organized and analyzed using Content Analysis technique in the thematic perspective with the theoretical support of the National Curriculum Guidelines for Dentistry Course (Diretrizes Curriculares Nacionais). The undergraduate students perceived the internship as an opportunity to experience the work reality in health services, developed and improved skills and competencies like communication, management and organization of their work, and construction of their professional identity. In conclusion, the students came closer to the concepts of health care, humanization, autonomy and learning at work, experiences that emerged as significant for them.

Descriptors: Education in Dentistry. Internships. Health Services. Humanization of Assistance.

\section{REFERÊNCIAS}

1. Brasil. Ministério da Saúde. Conselho Nacional de Secretarias Municipais de Saúde. Comparação entre sistemas de serviços de saúde da Europa e o SUS: pontos para reflexão da agenda da atenção básica: Relatório de Oficina da Rede Américas, Belém: CONASEMS; 2008.

2. Campos GWS, Amaral MA. A clínica ampliada e compartilhada, a gestão democrática e redes de atenção como referenciais teórico-operacionais para a reforma do hospital. Ciênc Saúde Coletiva. 2017;12(4):849-59.

3. Hayacibara MF, Lolli LF, Terada RSS, Hidalgo MM, Bispo CGC, Terada HH et al. Experiência de clínica ampliada em Odontologia na Universidade Estadual de Maringá. Rev Bras Educ Méd. 2012, 36(1Supl 2):178-83.

4. Mattos RA. Os sentidos da integralidade: algumas reflexões acerca de valores que merecem ser defendidos. In: Pinheiro R, Mattos RA. Os sentidos da integralidade na atenção e no cuidado à saúde. Rio de Janeiro: UERJ, IMS: ABRASCO, 2006. 184p.

5. Matos MS, Tenório R. Percepção de alunos, professores e usuários acerca da dimensão ética na formação de graduandos de odontologia. Ciênc Saúde Coletiva. 2010;15(Supl 2):3255-64.

6. Brasil. Ministério da Saúde. Portaria 198, de 13 de fevereiro de 2004. Institui a Política Nacional de Educação Permanente em Saúde para a formação e o desenvolvimento de trabalhadores para o setor e dá outras providências. Brasília; 2004.

7. Brasil. Ministério da Saúde. Portaria Interministerial $\mathrm{N}^{\mathrm{o}}-2.101$, de 3 de novembro de 2005. Institui o Programa Nacional de Reorientação da Formação Profissional em Saúde - Pró-Saúde - para os cursos de graduação em Medicina, Enfermagem e Odontologia. Brasília; 2005.

8. Brasil. Ministério da Saúde. Portaria interministerial $\mathrm{n}^{\circ} 1.802$, de 26 de agosto de 2008. Institui o programa de educação pelo trabalho para a saúde - PET - saúde. Ministério da Saúde, Brasília; 2008.

9. Sampaio J, Gomes LB. Potencialidades e desafios para a construção de redes colaborativas universidades - SUS: reflexões a partir da experiência da avaliação externa do PMAQ-AB. In: Gomes LB, Barbosa MG, Ferla AA. A educação permanente em saúde e as redes colaborativas: conexões para a produção de saberes e práticas. Porto Alegre: 
Rede UNIDA; 2016.

10. Mestriner SF, Sanches GL, Bulgarelli AF, Mestriner Jr W. Egressos do curso de odontologia: representações sociais de uma experiência extramuros. Health \& Social Change. 2014;5 (3):25-33.

11. Toassi RFC, Davoglio RS, Lemos VMA. Integração ensino-serviço-comunidade: o estágio na atenção básica da graduação em Odontologia. Educ Rev. 2012;28 (4):22342.

12. Mestriner Júnior W, Mestriner SF, Bulgarelli AF, Mishima SM. O desenvolvimento de competências em atenção básica à saúde: a experiência no projeto Huka-Katu. Ciênc Saúde Coletiva. 2011;16 (Suppl 1): 903-12.

13. Minayo MCS. O Desafio do Conhecimento: pesquisa qualitativa em saúde. 14ed., São Paulo: Hucitec; 2014.

14. Bondía JL. Notas sobre a experiência e o saber de experiência. Rev Bras Educ. 2002;19:20-8.

15. São Paulo. Diário Oficial do Estado de São Paulo, 2007; 117 (181): 43.

16. São Paulo. Diário Oficial do Estado de São Paulo, 2012; 122 (116): 42.

17. Brasil. Política nacional de saúde bucal. Diretrizes da Política Nacional de Saúde Bucal. Ministério da Saúde, Secretaria de Atenção à Saúde. Brasília, 2004b, 16p.

18. Brasil. Ministério da Educação e Conselho Nacional de Educação. Diretrizes Curriculares Nacionais dos Cursos de Graduação em Farmácia e Odontologia. Parecer CNE/CES 1.300/2001 - Despacho do Ministro em 4/12/2001, publicado no Diário Oficial da União de 7/12/2001, Seção 1, p. 25, Brasília, 2001.

19. Preti D, Rodrigues ACS. Análise de textos orais. v.1. FFLCH/USP. Projeto de Estudo da Norma Lingüística Urbana Culta de São Paulo; 1993.

20. Bardin, L. Análise de Conteúdo. Lisboa, Portugal: Edições 70, LDA; 2009.

21. Associação Brasileira de Ensino Odontológico. Diretrizes Curriculares Nacionais para os Cursos de graduação em Odontologia. Rev ABENO. 2002;2 (1):31-4.
22. Resolução CNS 466 de 12 de set 2012.Brasília, 2012. [Acesso em 1 set. 2016]. Disponível em: http://conselho. saude.gov.br/resolucoes/2012/Reso466.pdf

23. Leme PAT, Pereira AC, Meneghim MC, Mialhe FL. Perspectivas de graduandos em Odontologia acerca das experiências na atenção básica para sua formação em saúde. Ciênc Saúde Coletiva. 2015; 20(4): 125565.

24. Moimaz SAS, Saliba NA, Zina LG, Saliba O, Garbin CAS. Práticas de ensinoaprendizagem com base em cenários reais. Interface Comun Saúde Educ. 2010;14 (32):69-79.

25. Finkler, M, Caetano JC, Ramos FRS. Modelos, mercado e poder: elementos do currículo oculto que se revelam na formação em odontologia. Trab Educ Saúde. 2014;12(2):343-61.

26. Bulgarelli AF, Souza KR, Baumgarten AS, Souza JM, Rösing KC, Toassi RFC. Formação em saúde com vivência no Sistema Único de Saúde (SUS): percepções de estudantes do curso de Odontologia da Universidade Federal do Rio Grande do Sul (UFRGS), Brasil. Interface Comun Saúde Educ. 2014;18 (49):351-62.

27. Souza AL, Carcereri DL. Qualitative study of the teaching-service integration in an undergraduate Dentistry course. Interface Comunic Saúde Educ. 2011;15(39):107184.

28. Brondani MA, Komkham P, Jolanta A. How can dental public health competencies be addressed at the undergraduate level? J Public Health Dent. 2015;75(1):49-57.

29. Bulgarelli AF, Roperto RC, Mestriner SF, Mestriner W. Dentistry students' perceptions about an extramural experience with a Brazilian indigenous community. Indian $\mathbf{J}$ Dent Res. 2012; 23 (4):498-500.

30. Forte FDS, Pessoa TRRF, Freitas CHSM, Pereira CAL, Carvalho Junior PM. Reorientação na formação de cirurgiõesdentistas: o olhar dos preceptores sobre estágios supervisionados no Sistema Único de Saúde (SUS). Interface Comun Saúde 
Educ. 2015; 19 (Supl 1): 831-43.

31. Aguilar da Silva RH, Scapin LTP Batista, N. A. avaliação da formação interprofissional no ensino superior em saúde: aspectos da colaboração e do trabalho em equipe. Avaliação. 2011;16 (1):167-84.

32. Brasil. Ministério da Saúde. HumanizaSUS: Política Nacional de Humanização: a humanização como eixo norteador das práticas de atenção e gestão em todas as instâncias do SUS / Ministério da Saúde, Secretaria-Executiva, Núcleo Técnico da Política Nacional de Humanização. Brasília: Ministério da Saúde, 2004.

33. Mathew T, Shetty A, Shetty C, Narasimhan D, Shetty S, Hedge M. Comparison of communication skills between undergraduate dental students with and without prior training in effective communication. Nitte Univ J Health Sci. 2015;5(2):8.

34. Toassi RFC, Souza JM, Baumgarten A, Rosing CK. Avaliação curricular na educação superior em odontologia: discutindo as mudanças curriculares na formação em saúde no Brasil. Rev ABENO. 2012;12(2):170-7.

35. Silveira JLGC, Garcia VL. Mudança curricular em Odontologia: significados a partir dos sujeitos da aprendizagem. Interface Comun Saúde Educ. 2015;19 (52):145-58.

36. Morita MC, Kriger L, Carvalho ACP, Haddad AE. Implantação das Diretrizes Curriculares Nacionais em Odontologia. 1a ed. Maringá: Dental Press, ABENO, OPAS, MS; 2007.
37. Almeida M. Diretrizes curriculares nacionais para os cursos universitários da área da saúde. 2a ed. Londrina: Rede Unida; 2005.

38. Finkler M, Verdi MIM, Caetano JC, Ramos FRS. Formação profissional ética: um compromisso a partir das diretrizes curriculares? Trab Educ Saúde. 2010; 8(3) :449-62.

39. Lamers JMS, Baumgarten A, Bitencourt FV, Toassi RFC. Mudanças curriculares na educação superior em Odontologia: inovações, resistências e avanços conquistados. Rev ABENO. 2016;16(4) :2-18.

40. Peduzzi M, Del Gerral EA, Braga CP, Lucena FS, da Silva JAM. Atividades educativas de trabalhadores na atenção primária: concepções de educação permanente e de educação continuada em saúde presentes no cotidiano de Unidades Básicas de Saúde em São Paulo. Interface Comun Saúde Educ. 2009;13(30):121-34.

41. Batista KBC, Gonçalves OSJ. Formação dos profissionais de saúde para o SUS: significado e cuidado. Saúde Soc. 2011; 20(4):884-99.

Correspondência para:

Soraya Fernandes Mestriner

e-mail: somestri@ forp.usp.br

Avenida do Café s/ $n^{\circ}$, Campus da USP

14040-904 - Ribeirão Preto/SP 